\title{
Colossal magnetocapacitance and scale-invariant dielectric response in phase-separated manganites
}

\author{
RYAN P. RAIRIGH, GUNEETA SINGH-BHALLA, SEFAATIN TONGAY, TARA DHAKAL, AMLAN BISWAS \\ AND ARTHUR F. HEBARD*
}

Department of Physics, University of Florida, Gainesville, Florida 32611-8440, USA

*e-mail: afh@phys.ufl.edu

A full characterization of phase separation and the competition between phases is necessary for a comprehensive understanding of strongly correlated electron materials, such as under-doped high-temperature superconductors ${ }^{1,2}$, complex oxide heterojunctions ${ }^{3}$, spinels $s^{4,5}$, multiferroics ${ }^{6,7}$, rare-earth ferroelectric manganites ${ }^{8}$ and mixed-valence manganites in which phase competition is the dominant mechanism governing the insulator-metal (IM) transition and the associated colossal magnetoresistance effect $^{9-11}$. Thin films of strongly correlated electron materials are often grown epitaxially on planar substrates and typically have anisotropic properties that are usually not captured by edge-mounted four-terminal electrical measurements, which are primarily sensitive to inplane conduction paths. We present an experimental study of anisotropic transport in phase-separated manganite thin films by using an unconventional arrangement of contact electrodes that enables the simultaneous determination of colossal magnetoresistance associated with d.c. transport parallel to the film substrate and colossal magnetocapacitance associated with a.c. transport in the perpendicular direction. We distinguish two distinct direction-dependent IM transitions and find a dielectric response that collapses onto a scale-invariant dependence over a large range of frequency, temperature and magnetic field.

To demonstrate our technique, we use a 600 - $\AA$-thick film of the phase-separated manganite $\left(\mathrm{La}_{1-y} \mathrm{Pr}_{y}\right)_{0.67} \mathrm{Ca}_{0.33} \mathrm{MnO}_{3}$ (LPCMO) as the base electrode in a metal-insulator-metal (MIM) trilayer capacitor structure. The dielectric response is characterized by Cole-Cole plots. The phase diagram over which collapse occurs defines an extended region where the competing interaction of the coexisting ferromagnetic metal (FMM) and charge-ordered insulator (COI) phases $^{10-15}$ has the same behaviour over a wide range of temporal and spatial scales. At low frequency, corresponding to long length scales, the volume of the phase diagram collapses to a point defining the zero-field IM percolation transition in the perpendicular direction.

The simultaneous measurement of the zero-field $(H=0)$ parallel resistance, $R_{\|}(T)$, and perpendicular capacitance, $C(T)$, in the LPCMO film, which is the base electrode in the trilayer configuration shown schematically in Fig. 1a, is captured in the temperature-dependent curves of Fig. 1b. The two-terminal $C(T)$ measurements correspond to the real part, $C^{\prime}(\omega)$, of a complex lossy capacitance, $C^{*}(\omega)=C^{\prime}(\omega)-i C^{\prime \prime}(\omega)$, measured at a frequency $f=\omega / 2 \pi=0.5 \mathrm{kHz}$. As temperature, $T$, decreases from $300 \mathrm{~K}$, the prevailing high-temperature paramagnetic-insulator phase gives way near $T=220 \mathrm{~K}$ (ref. 14) to a dominant COI phase. Minority-phase FMM domains appear and begin to short circuit the resistance rise as $T$ continues to decrease. At the resistance peaks, the percolative IM transition for transport in the parallel direction through FMM domains occurs at temperatures $T_{\mathrm{IM}, \|}^{\downarrow}=95 \mathrm{~K}$ and $T_{\mathrm{IM}, \|}^{\uparrow}=106 \mathrm{~K}$, where $\downarrow / \uparrow$ indicate the cooling/warming direction of the temperature sweep. Below $T_{\text {IM, }}^{\downarrow}$, the FMM phase rapidly dominates with decreasing $T$, and $R_{\|}$ decreases by four orders of magnitude.

The $C(T)^{\downarrow \uparrow}$ traces reach plateaux at high and low temperature where the LPCMO is in its respective paramagnetic-insulator and FMM states, both of which have sufficiently low resistivity to act as metallic electrodes in a MIM structure. Between the $C_{\mathrm{AlOx}}(T)$ plateaux, the $C(T)^{\downarrow \uparrow}$ traces show capacitance minima $\sim 20 \mathrm{~K}$ below the corresponding resistance maxima. At the perpendicular field $H=50 \mathrm{kOe}$, the capacitance (blue curve) has increased by a factor of 1,000 above the zero-field minimum; colossal magnetocapacitance is clearly present. The remnant capacitance dip at $50 \mathrm{kOe}$ disappears at $70 \mathrm{kOe}$ and the linear temperature dependence, $C(T)=C_{\mathrm{AlOx}}(T)$, is identical to that of separately measured $\mathrm{Al} / \mathrm{AlOx} / \mathrm{Al}$ capacitors. Deviations of $C(T)^{\downarrow \uparrow}$ below $C_{\text {AlOx }}(T)$ thus reflect the competition of FMM and COI phases, and it is here that the colossal magnetocapacitance effects occur.

To understand how measurement of $C(T)$ is not a complicated way of measuring $R(T)$, we analyse in detail circuit equivalents (see the Supplementary Information) of the measurement configuration. We start by modelling the measurement configuration as a resistance, $R_{\mathrm{s}}$, in series with the parallel combination of $C^{*}(\omega)$ and a d.c. resistance, $R_{0}$. We then justify this model by establishing two necessary and sufficient conditions to assure that $R_{\mathrm{s}}$ can be ignored, namely: (1) the leakage resistance, $R_{0}$, is significantly higher than any of the impedance elements in our configuration, and (2) frequencies are chosen such that $R_{\mathrm{s}}$ is the smallest relevant impedance. Under these conditions the measured quantity, $C^{*}(\omega)$, is determined by voltage drops that are perpendicular to the film interface and the corresponding inplane equipotentials shown schematically by the dashed horizontal lines in the circuit schematic diagram of Fig. 1c.

We model $C^{*}(\omega)$ using a Maxwell-Wagner circuit equivalent ${ }^{16}$ (see the Supplementary Information) in which the manganite 
a

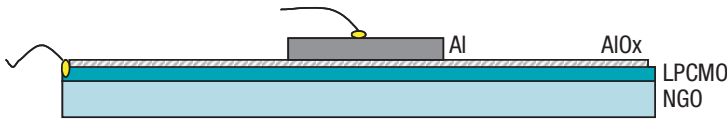

b

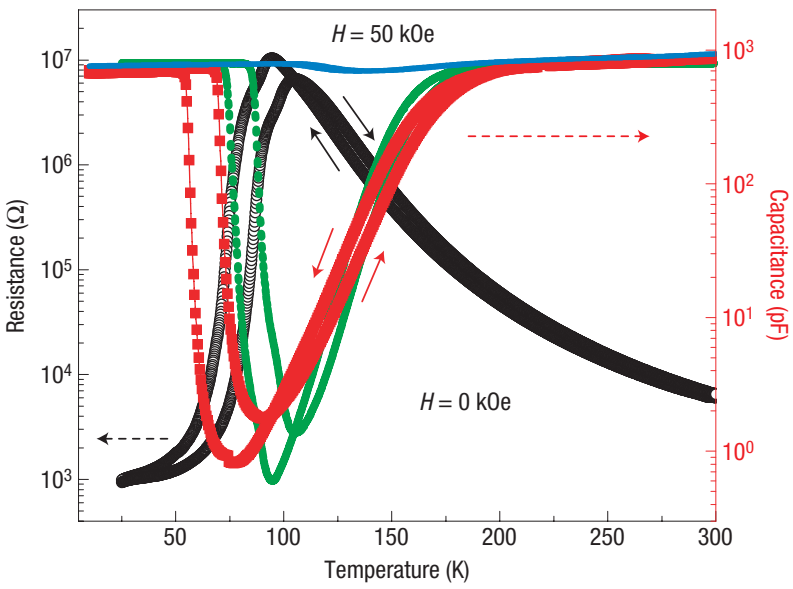

G

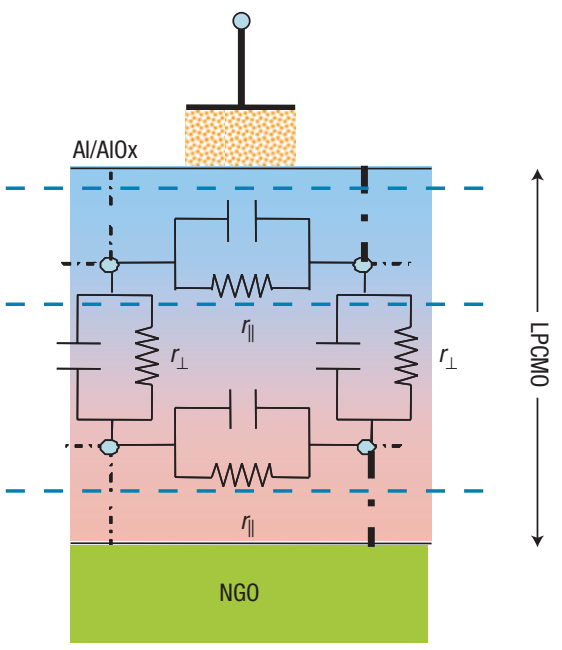

Figure 1 Parallel (resistance) and perpendicular (capacitance) transport measurements are made on the same LPCMO film. a, Cross-sectional schematic view of the trilayer capacitor structure comprising the LPCMO base electrode, the AlOx dielectric and the Al counter electrode. $\mathbf{b}$, Semi-logarithmic plots of the temperature-dependent resistances, $R_{\|}^{\downarrow \uparrow}(T)$ (black), and capacitances, $C_{\perp}^{\downarrow \uparrow}(T)$ (red), for decreasing $(\downarrow)$ and increasing $(\uparrow)$ temperatures at zero field obtained on the same structure. The thin green lines are fits to the Maxwell-Wagner model (see the Supplementary Information) which incorporates $R_{\|}^{\downarrow \uparrow}(T)$ as an input and therefore produces capacitance minima at temperatures coincident with the resistance maxima. At $50 \mathrm{kOe}$ (blue), the capacitance has increased from the zero-field minima by a factor of 1,000. c, Schematic representation of the LPCMO film using distributed circuit elements. Strain effects give rise to a resistance gradient in the perpendicular direction represented schematically by an unequal spacing of equipotentials (dashed horizontal lines) superimposed on a colour gradient.

impedance, expressed as a parallel combination of a resistance $R_{\mathrm{M}}$ and capacitance $C_{\mathrm{M}}$, is connected in series with a leak-free capacitance, $C_{\mathrm{AlOx}}$, representing the $\mathrm{Al} / \mathrm{AlOx}$ circuit element. The qualitative agreement shown in Fig. $1 \mathrm{~b}$ between the capacitance data (red) and the Maxwell-Wagner model calculation (green) confirms the appropriateness of the Maxwell-Wagner model as has also been shown in related dielectric studies of transition-metal oxides $^{17}$, spinels $s^{4,5,18}$ and multiferroics ${ }^{7}$ where the material under investigation is the 'insulator' (I) of a MIM structure rather than the base electrode as discussed here.

The Maxwell-Wagner analysis uses the measured $R_{\|}$as an input and therefore enforces an alignment in temperature (Fig. 1b) between the measured resistance maxima and the calculated capacitance minima. As the equipotentials of the capacitance measurement are parallel to the film surface, the misalignment of the measured capacitance minima $(\sim 20 \mathrm{~K}$ in Fig. $1 \mathrm{~b})$ can best be explained by assuming that $R_{\mathrm{M}}$ in the Maxwell-Wagner calculation should be the perpendicular resistance, $R_{\perp}(T)$, rather than $R_{\|}(T)$. Hence the measured capacitance minima are in alignment with putative resistance maxima corresponding to percolative IM transitions in $R_{\perp}(T)$. The IM transition temperatures in the perpendicular direction, $T_{\mathrm{IM}, \perp}^{\downarrow}=77 \mathrm{~K}$ and $T_{\mathrm{IM}, \perp}^{\uparrow}=91 \mathrm{~K}$, are therefore equal to the temperatures where the capacitance minima occur at noticeably lower values than the corresponding temperatures for the $R_{\|}(T)$ maxima.

Strain effects explain the two separate percolation transitions. The LPCMO films were grown on $\mathrm{NdGaO}_{3}$, which is known to stabilize the pseudocubic structure of the FMM phase at low temperatures $^{19,20}$. The effect of the substrate diminishes away from the interface ${ }^{19}$ as shown schematically by the shading in Fig. 1c, where the LPCMO electrode is shown as an infinite resistor-capacitor network comprising local resistances $r_{\perp}$ and $r_{\|}$respectively perpendicular and parallel to the interface. These distributed resistor elements are locally equal to each other but increase as a function of distance away from the interface ${ }^{21}$. In the measurement of $R_{\|}$, the strain-stabilized FMM region 'shorts out' the higher resistance state occurring away from the interface, and percolation occurs at a higher temperature $T_{\mathrm{IM}, \|}^{\downarrow \uparrow}$ than $T_{\mathrm{IM}, \perp}^{\downarrow \uparrow}$ as measured by the capacitance minima. For thicker films the two IM transitions converge, as experimentally confirmed over the thickness range 300-900 (see the Supplementary Information), to a single value representing isotropic threedimensional percolation of bulk LPCMO with the temperature difference $\Delta T_{\mathrm{IM}}^{\downarrow \uparrow}=T_{\mathrm{IM}, \|}^{\downarrow \uparrow}-T_{\mathrm{IM}, \perp}^{\downarrow \uparrow}$ approaching zero.

To fully characterize the intrinsic dielectric response of the LPCMO film, we use Cole-Cole plots in which $C^{\prime \prime}$, proportional to dielectric loss, is presented as a function of $C^{\prime}$, proportional to the real part of the dielectric constant, while an external parameter, usually frequency, is varied ${ }^{16}$. We show such a plot on logarithmic axes in Fig. $2 \mathrm{a}$ at $T=65 \mathrm{~K}$ (warming) for the magnetic fields indicated in the inset. As the frequency is swept from low $(50 \mathrm{~Hz})$ to high $(20 \mathrm{kHz})$ at each field, $C^{\prime \prime}(\omega)$ passes from a region where $C_{\mathrm{AlOx}}$ dominates, subsequently reaches a peak value at $\omega R_{\mathrm{M}} C_{\mathrm{M}}=1$ (vertical arrow), and then enters the high-frequency region where the intrinsic response of the manganite dominates and the data collapse onto the same curve. The low-to-high frequency crossover from no-collapse to collapse is magnified in the inset.

At higher temperatures, the data collapse is more striking as shown in the Cole-Cole plot of Fig. 2b. Independently of whether the implicit variable is $f=\omega / 2 \pi, T$ or $H$, the dielectric response collapses onto the same curve when the remaining two variables are fixed (see inset). As $\omega$ increases, or equivalently, as $T$ or $H$ decrease, $C^{\prime \prime}$ approaches zero and $C^{\prime}$ approaches a constant, $C_{\infty}$, representing the bare dielectric response. We find that when $C^{\prime \prime}$ is plotted against 

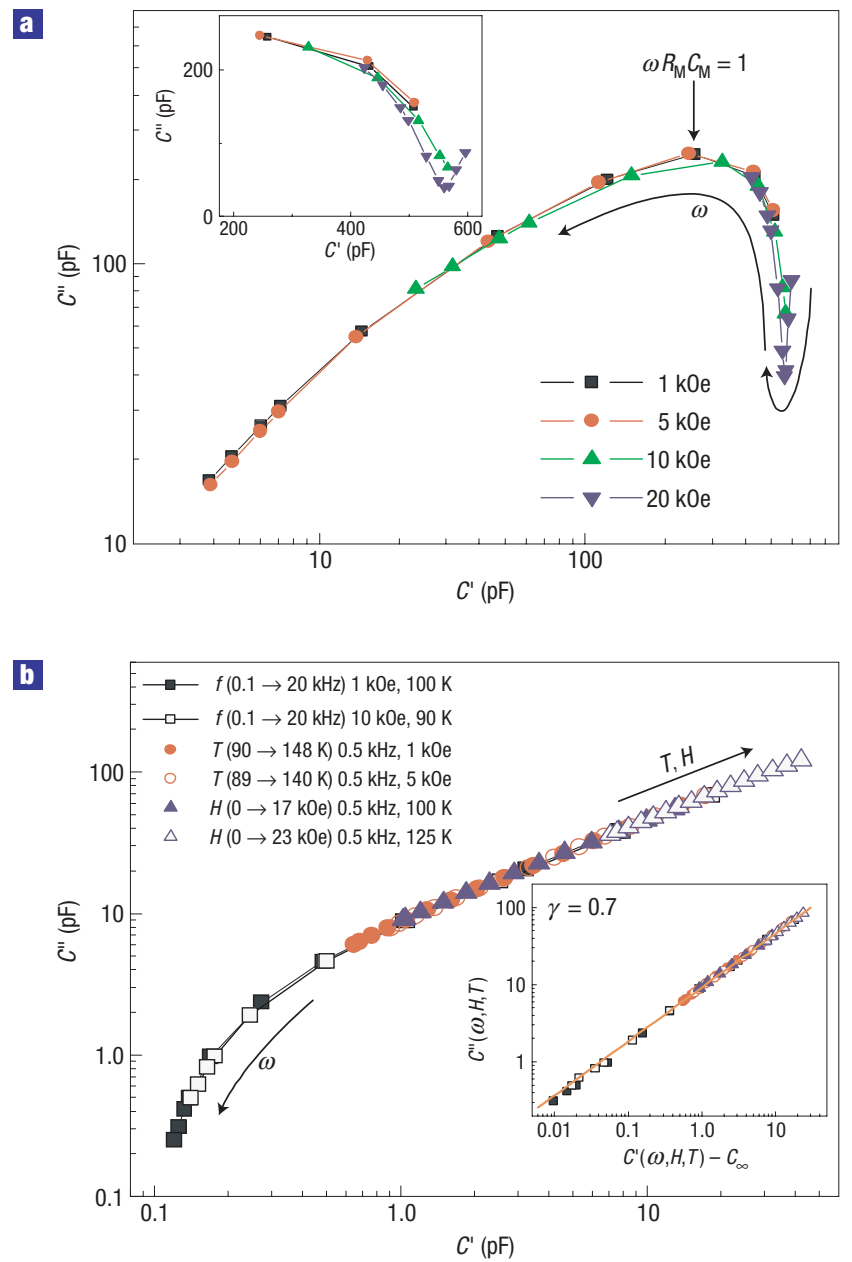

Figure 2 Cole-Cole plots reveal data collapse and power-law scaling of the dielectric response. a, Cole-Cole plot on logarithmic axes of dielectric dissipation, $C^{\prime \prime}(\omega)$, versus polarization, $C^{\prime}(\omega)$, at $T=65 \mathrm{~K}$ (warming) for the magnetic fields indicated in the legend. At each field, the implicit frequency variable, $f$, ranges from $100 \mathrm{~Hz}$ to $20 \mathrm{kHz}$. The crossover from non-overlapping traces at low frequencies to data collapse at high frequencies is magnified in the inset. $\mathbf{b}$, Cole-Cole plot for the sweep parameters and ranges indicated in the legend. Independently of whether the implicit variable is frequency, temperature or magnetic field, the data collapse onto the same curve regardless of the parameter being varied. These same data, replotted as a straight orange line in the inset after subtracting a single fitting parameter, $C_{\infty}$, show PLSC.

$C^{\prime}-C_{\infty}$ on double logarithmic axes, all of the data collapse onto a straight line (inset) described by the equation,

$$
C^{\prime \prime}(\omega, T, H)=\Lambda\left[C^{\prime}(\omega, T, H)-C_{\infty}\right]^{\gamma} .
$$

The three fitting parameters have values $\Lambda=9.404 \pm 0.049 \mathrm{pF}^{0.3}$, $\gamma=0.701 \pm 0.004$ and $C_{\infty}=0.135 \pm 0.003 \mathrm{pF}$. We note that equation (1) is a generalization of Jonscher's 'universal dielectric response', which requires $\gamma=1$ and describes well the highfrequency dielectric response of most dielectrics ${ }^{22}$. As shown below, the generalized power-law scaling that we have observed with respect to three independent variables $(\omega, T, H)$ is associated with a percolation transition in which FMM and COI phases form clusters that compete on self-similar length scales.

The universal power-law scaling collapse (PLSC) of the data described by equation (1) applies to a region of $f T H$ space with

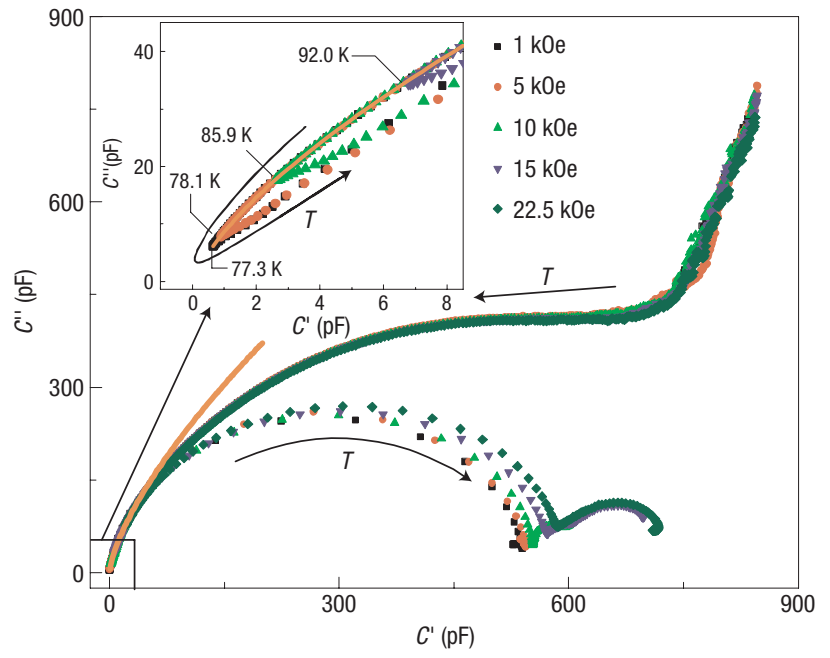

Figure 3 Determination of the boundaries of the PLSC region. Cole-Cole plot on linear axes with temperature (cooling) as the implicit variable for $f=500 \mathrm{~Hz}$ and magnetic fields identified in the legend. The well-defined transitions onto/(off of) the orange PLSC line (magnified in the inset), which is the same line as that shown in the inset of Fig. $2 b$, define respectively the upper/lower field-dependent critical temperatures that bound the PLSC behaviour in fTH space shown in Fig. 4.

boundaries that can be accurately determined from Cole-Cole plots that use $T$ as the implicit variable over the entire temperature range. As an example of our procedure, in Fig. 3 we show a Cole-Cole plot on linear axes with temperature (cooling) as the implicit variable for $f=500 \mathrm{~Hz}$ and magnetic fields identified in the legend. The dielectric response is divided into two branches by a pronounced crossover from PLSC behaviour denoted by the orange line described by equation (1) (see also Fig. 2b inset) in common with the lower part of the upper branch to no-collapse (NC) behaviour (lower branch). As temperature decreases on the upper branch, the data follow a non-power-law collapse (NPLC) and at well-defined field-dependent upper critical temperatures, $T_{\text {upper }}^{\downarrow}(H)$, merge onto the PLSC line. As $T$ continues to decrease, there is a second critical temperature, $T_{\text {lower }}^{\downarrow}(H)$, marking the demarcation points between branches by an almost $180^{\circ}$ change in the direction of the trajectory as indicated by the 'turnaround' arrow in the inset. These field-dependent crossover points precisely define $T_{\text {lower }}^{\downarrow}(H)=T_{C \min }^{\downarrow}(H)$, where $C^{\prime}(H)$ is minimum and which by our Maxwell-Wagner analysis has been shown to be the same as $T_{\mathrm{IM}, \perp}^{\downarrow}(H)$. A similar analysis holds for warming curves.

The accurate determination of field-dependent criticaltemperature boundaries at each frequency allows us to delineate triangular-shaped areas in TH space where PLSC is obeyed as shown for cooling in Fig. 4. The two critical-temperature boundaries, $T_{\text {upper }}^{\downarrow}(H)$ and $T_{\text {lower }}^{\downarrow}(H)$, are determined by the NPLCto-PLSC (filled symbols) and the PLSC-to-NC transition (open symbols). For comparison, we show (crosses) the IM boundary $T_{\mathrm{IM}, \|}^{\downarrow}(H)$, determined from the peaks in $R_{\|}^{\downarrow}(H)$. The roughly parallel offset from the lower boundary $T_{\mathrm{IM}, \perp}^{\downarrow}(H)$ of the PLSC phase implies separate percolation transitions for $R_{\perp}$ and $R_{\|}$. Increasing the frequency pushes $T_{\text {upper }}^{\downarrow}(H)$ to higher temperatures and fields, thus increasing the volume of $f T H$ space where PLSC occurs. For any $T H$ region bounded by an upper critical boundary determined at $f=f_{0}$, PLSC is obeyed within that region for all $f \geq f_{0}$. Therefore, as $f_{0}$ decreases and the corresponding length scales being probed increase, the corresponding $T H$ region where 


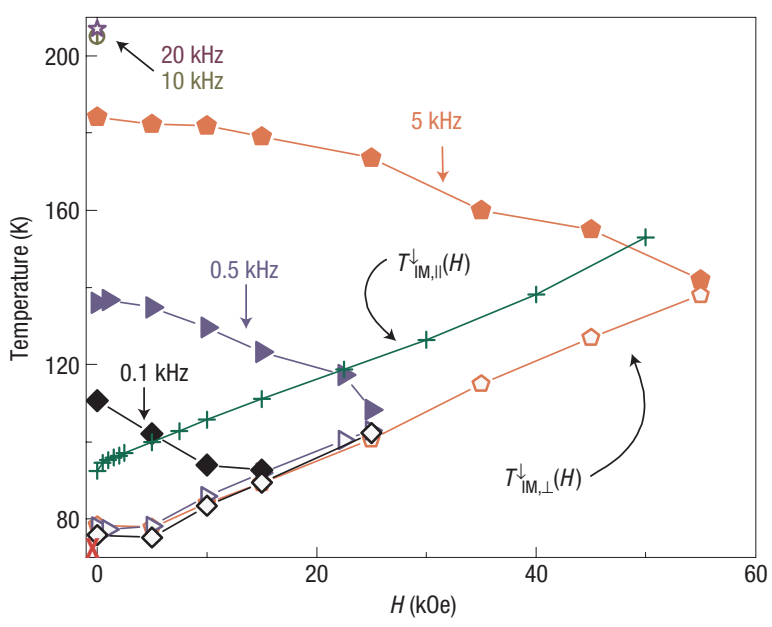

Figure 4 PLSC holds over a wide region of frequency-temperature-field (fTH) space and at low frequency converges to a point defining the IM transition, $\boldsymbol{T}_{\mathrm{IM}, \perp}^{\downarrow}(\boldsymbol{H}=\mathbf{0})$. The determination of upper (filled symbols) and lower (open symbols) critical-temperature lines (cooling, Fig. 3) define triangular-shaped regions in $T H$ space within which PLSC holds for frequencies higher than the frequency (labelled) at which the upper boundary is determined. As $f$ decreases, the PLSC region shrinks to a point (large red $X)$ that marks $T_{\mathrm{IM}, \perp}^{\downarrow}(H=0)$ and anchors the lower critical-temperature line, $T_{\mathrm{IM}, \perp}^{\downarrow}(H)$, where the capacitance is minimum. The critical temperature line, $T_{\text {IM. } \|}^{\downarrow}(H)$ (green), determined from the peaks in $R_{\|}^{\downarrow}(H)$ is roughly parallel to $T_{\mathrm{IM}, \perp}^{\downarrow}(H)$ at a temperature offset by $\sim 20 \mathrm{~K}$.

PLSC is obeyed shrinks to a point defined by the temperature $T_{\mathrm{IM}, \perp}^{\downarrow}=77 \mathrm{~K}$ at $H=0$ (marked with a red $\mathrm{X}$ in Fig. 4) where $R_{\perp}$ is maximum and percolation occurs. A similar phase diagram shifted $\sim 20 \mathrm{~K}$ to higher temperatures occurs for warming data.

The notion of percolative phase separation in mixed-phase manganites ${ }^{10,23}$ recognizes that the percolation transition as a function of temperature is different from the classic percolation problem that deals with connectivity occurring as a function of composition rather than temperature. This complication leads to different identifications of the percolation transition temperature in manganites determined, for example, by noise measurements ${ }^{23,24}$. As the phenomenology of percolation incorporates power-law scaling descriptions ${ }^{25}$ similar to those described in this paper, we are confident in identifying $T_{\mathrm{IM}, \perp}^{\downarrow \uparrow}$, which coincides with the maximum in $R_{\perp}(T)$ and about which PLSC occurs, as the percolation transition temperature.

We expect our measurement technique, which advantageously includes information about dynamics, will find wide application in studies of a variety of anisotropic thin-film systems, including those where the presence of competing phases is under debate. Candidates for study include layered manganites ${ }^{26}$, under-doped high- $T_{\mathrm{c}}$ superconductors ${ }^{1}$, and electron-doped cuprates $^{27}$ and $c$-axis graphite with anisotropic resistivity ratios $\left(\rho_{c} / \rho_{a b}\right)$ reported to be as high as 10,000 (ref. 28) and 1,000 (ref. 29) respectively. We have also demonstrated that our technique can be sensitive to strain at epitaxial interfaces and thus capable of determining, through thickness dependence studies, the critical thickness for a relaxed top interface. By incorporating a LPCMO film as the 'metal' (M) base electrode of a MIM structure and using an ultralow-leakage dielectric ( $\mathrm{AlOx}$ ) for the insulating spacer, we prevent the metallic phase of the LPCMO from shorting out the measurement as it would if it were the middle layer (I) of a conventional dielectric configuration. Circuit analysis shows, and experiment demonstrates, that over a well-defined frequency range, the large $\mathrm{Al} / \mathrm{AlOx}$ capacitor acts as a baseline reference that 'decloaks' or makes visible the much smaller capacitance of the series-connected LPCMO film.

In summary, by distinguishing two strain-related directiondependent IM transitions, we confirm the sensitivity of our capacitance and resistance measurements to perpendicular and longitudinal transport and simultaneously establish phase diagrams in $f T H$ space for $T>T_{\mathrm{IM}, \perp}^{\downarrow \uparrow}(H)$ where PLSC is observed. The power-law dependence implies scale invariance from shortlength high-frequency scales to significantly longer length scales, where the low-frequency PLSC regions collapse to the points $T_{\mathrm{IM}, \perp}^{\downarrow \uparrow}(H=0)$ defining the d.c. cooling/warming percolation transitions. The factor of 1,000 change in capacitance is too large to be caused by changes on the surfaces of the LPCMO and must therefore be attributed to the competition of microscopic FMM/COI clusters intrinsic to the bulk manganite. In addition, to sustain a resistance gradient in the perpendicular direction, the shortest length scale in the PLSC region must be smaller than the LPCMO film thickness $(600 \AA)$ ). As temperature is lowered through $T_{\mathrm{IM}, \perp}^{\downarrow \uparrow}(H)$, the competition of phases on microscopic $(\sim 100 \AA)$ length scales in the PLSC region crosses over to a competition on macroscopic $(\sim 1 \mu \mathrm{m})$ length scales in which the metallic fraction of area covered increases rapidly from $C_{\min } / C_{\mathrm{AlOx}}=10^{-3}$ to unity as large clusters of the FMM phase dominate ${ }^{10,11}$. A full understanding of these results will be a challenge to the contrasting theories of phase separation and competition in manganites based on intrinsic disorder ${ }^{12}$, long-range strain interactions ${ }^{13}$, blocked metastable states ${ }^{14}$ or thermally equilibrated electronically soft phases ${ }^{15}$.

\section{METHODS}

The 600- $\AA$-thick $\left(\mathrm{La}_{1-y} \mathrm{Pr}_{y}\right)_{1-x} \mathrm{Ca}_{x} \mathrm{MnO}_{3}$ (LPCMO) thin films were grown at a stoichiometry of $x=0.33$ and $y=0.5$. The value of $x$ was chosen to maximize the Curie temperature of the FMM phase. The value $y=0.5$ was chosen because at this composition thin films of LPCMO show a large hysteresis in resistivity in the cooling and warming cycles, which is a signature of phase coexistence. For thin films with $y<0.4$ the hysteresis becomes negligible, whereas for $y>0.6$ the high resistance at $T_{\mathrm{IM}}$ requires extremely low leakage resistance of the $\mathrm{AlOx}$ dielectric. The films were grown using pulsed laser deposition at a rate of $0.05 \mathrm{~nm} \mathrm{~s}^{-1}$ on $\mathrm{NdGaO}_{3}$ (NGO) (110) substrates kept at $820^{\circ} \mathrm{C}$ in an oxygen atmosphere of $420 \mathrm{mtorr}$. Standard $\theta-2 \theta \mathrm{X}$-ray diffraction data show that the films are epitaxial and of a single chemical phase.

The $100-\AA$-thick AlOx dielectric was deposited at a rate of $2-3 \AA \mathrm{min}^{-1}$ by radiofrequency magnetron sputtering of an alumina target using separate ex situ procedures previously described ${ }^{30}$. A critical step to assure a leak-free insulating AlOx layer is the preconditioning of the alumina target in a partial pressure of oxygen before sputter deposition in pure argon ambient. The $1,000-\AA$-thick circular $\mathrm{Al}$ counter electrode with radius $0.4 \mathrm{~mm}$ was thermally evaporated through a shadow mask in a separate chamber.

The capacitance measurements (Fig. 1b, red) were made using an Andeen-Hagerling AH2700A capacitance bridge in a guarded three-terminal mode at stepped frequencies ranging from $50-20,000 \mathrm{~Hz}$. Two of the terminals were connected to the sample leads shown schematically in Fig. 1a and the third to an electrically isolated copper can surrounding the sample and connected to the ground of the bridge circuit. Most of the measurements were made using $25 \mathrm{mV}$ r.m.s. excitation, and linearity was confirmed at all fields and temperatures. The bridge was set to output data in the parallel mode in which the sample is assumed to be the circuit equivalent of a capacitance $C^{\prime}$ in parallel with a resistance $R$ (see the Supplementary Information).

Contacts to the sample were made to the base LPCMO film using pressed indium and to the $\mathrm{Al}$ counter electrode using fine gold wire held in place with silver paint. Silver paint was also occasionally used to make contact to the base electrode with no consequence to the capacitance data at all measurement frequencies. The series combination of the LPCMO parallel resistance with the leakage resistance of the $\mathrm{AlOx}$ dielectric was found to be immeasurably large with a lower bound $>10^{10} \Omega$ determined by replacing the sample with a standard $10^{10} \Omega$ ceramic resistor. Thus the leakage resistance is more than a 
factor of 1,000 higher than the $\sim 10 \mathrm{M} \Omega$ maximum resistance of our LPCMO film (Fig. 1b).

The four-terminal resistance measurements of d.c. transport parallel to the film interfaces (Fig. 1b, black) were made using evenly spaced Van der Pauw contacts (not shown in Fig. 1a) directly connected at the LPCMO film edges. To check for any frequency dependence in the parallel resistance of the LPCMO and associated contact resistance, we carried out a two-terminal a.c. measurement by applying a sinusoidal voltage to all pairs of the LPCMO contacts and used a lock-in amplifier to synchronously detect the output of a wide-band current amplifier that provided a return path to ground for the sample current. The two-terminal resistance was similar to the four-terminal measurement. Importantly, no frequency dependence in the range $50-20,000 \mathrm{~Hz}$ for the resistance varying from $1 \mathrm{k} \Omega$ to $20 \mathrm{M} \Omega$ (Fig. 1b) was detected, thus assuring that all of the frequency dependence seen in the capacitance measurement is due to perpendicular rather than parallel transport. In addition, we established in separate experiments on symmetric $\mathrm{Al} / \mathrm{AlOx} / \mathrm{Al}$ structures that $C_{\mathrm{AlOx}}$ has negligible frequency dispersion over the same frequency range.

Received 19 December 2006; accepted 1 May 2007; published 27 May 2007.

\section{References}

1. Oh, S., Crane, T. A., Van Harlingen, D. J. \& Eckstein, J. N. Doping controlled superconductor-insulator transition in $\mathrm{Bi}_{2} \mathrm{Sr}_{2-x} \mathrm{La}_{x} \mathrm{CaCu}_{2} \mathrm{O}_{8+d}$. Phys. Rev. Lett. 96, 107003 (2006).

2. Lang, K. M. et al. Imaging the granular structure of high- $T_{\mathrm{c}}$ superconductivity in underdoped $\mathrm{Bi}_{2} \mathrm{Sr}_{2} \mathrm{CaCu}_{2} \mathrm{O}_{8+\mathrm{d}}$. Nature 415, 412-416 (2002).

3. Nakagawa, N., Asai, M., Mukunoki, Y., Susaki, T. \& Hwang, H. Y. Magnetocapacitance and exponential magnetoresistance in manganite-titanate heterojunctions. Appl. Phys. Lett. 86, $082504(2005)$

4. Weber, S. et al. Colossal magnetocapacitance and colossal magnetoresistance in $\mathrm{HgCr}_{2} \mathrm{~S}_{4}$. Phys. Rev Lett. 96, 157202 (2006).

5. Hemberger, J. et al. Relaxor ferroelectricity and colossal magnetocapacitive coupling in ferromagnetic $\mathrm{CdCr}_{2} \mathrm{~S}_{4}$. Nature 434, 364-367 (2005)

6. Singh, M. P., Prellier, W., Mechin, L. \& Raveau, B. Effect of ferroelectric layers on the magnetocapacitance properties of superlattices-based oxide multiferroics. Appl. Phys. Lett. $\mathbf{8 8}$ 012903 (2006).

7. Catalan, G. Magnetocapacitance without magnetoelectric coupling. Appl. Phys. Lett. 88, 102902 (2006).

8. Goto, T., Kimura, T., Lawes, G., Ramirez, A. P. \& Tokura, Y. Ferroelectricity and giant magnetocapacitance in perovskite rare-earth manganites. Phys. Rev. Lett. 92, 257201 (2004).

9. Moreo, A., Yunoki, S. \& Dagotto, E. Solid state physics-Phase separation scenario for manganese oxides and related materials. Science 283, 2034-2040 (1999).

10. Uehara, M., Mori, S., Chen, C. H. \& Cheong, S.-W. Percolative phase separation underlies colossal magnetoresistance in mixed-valent manganites. Nature 399, 560-563 (1999).
11. Zhang, L. W., Israel, C., Biswas, A., Greene, R. L. \& de Lozanne, A. Direct observation of percolation in a manganite thin film. Science 298, 805-807 (2002).

12. Burgy, J., Moreo, A. \& Dagotto, E. Relevance of cooperative lattice effects and stress fields in phase-separation theories for CMR manganites. Phys. Rev. Lett. 92, 097202 (2004).

13. Ahn, K. H., Lookman, T. \& Bishop, A. R. Strain-induced metal-insulator phase coexistence in perovskite manganites. Nature 428, 401-404 (2004).

14. Ghivelder, L. \& Parisi, F. Dynamic phase separation in $\mathrm{La}_{5 / 8-y} \mathrm{Pr}_{y} \mathrm{Ca}_{3 / 8} \mathrm{MnO}_{3}$. Phys. Rev. B 71, 184425 (2005).

15. Milward, G. C., Calderon, M. J. \& Littlewood, P. B. Electronically soft phases in manganites. Nature 433, 607-610 (2005).

16. Jonscher, A. K. Dielectric Relaxation in Solids (Chelsea Dielectrics, London, 1983).

17. Lunkenheimer, P. et al. Origin of apparent colossal dielectric constants. Phys. Rev. B 66, 052105 (2002).

18. Lunkenheimer, P., Fichtl, R., Hemberger, J., Tsurkan, V. \& Loidl, A. Relaxation dynamics and colossal magnetocapacitive effect in $\mathrm{CdCr}_{2} \mathrm{~S}_{4}$. Phys. Rev. B 72, 060103 (2005).

19. Sun, J. Z., Abraham, D. W., Rao, R. A. \& Eom, C. B. Thickness-dependent magnetotransport in ultrathin manganite films. Appl. Phys. Lett. 74, 3017-3019 (1999).

20. Biswas, A. et al. Strain-driven charge-ordered state in $\mathrm{La}_{0.67} \mathrm{Ca}_{0.33} \mathrm{MnO}_{3}$. Phys. Rev. B 63, 184424 (2001).

21. Petit, M. et al. Depth analysis of the in-plane lattice constants in compressively strained $\mathrm{La}_{0.67} \mathrm{Ca}_{0.33} \mathrm{MnO}_{3}$ thin films. J. Appl. Phys. 97, 093512 (2005).

22. Jonscher, A. K. The 'Universal' dielectric response. Nature 267, 673-679 (1977).

23. Han, K.-H., Huang, Q., Ong, P. C. \& Ong, C. K. Thermal hysteresis in low-frequency noise of $\mathrm{La}_{0.7} \mathrm{Sr}_{0.3} \mathrm{Mn}_{0.92} \mathrm{Fe}_{0.08} \mathrm{O}_{3}$ thin films at low magnetic field. J. Phys. Condens. Matter 13, $8745-8753(2001)$.

24. Podzorov, V., Uehara, M., Gershenson, M. E., Koo, T. Y. \& Cheong, S.-W. Giant 1/f noise in perovskite manganites: Evidence of the percolation threshold. Phys. Rev. B 61, R3784 (2000).

25. Stauffer, D. \& Aharony, A. Introduction to Percolation Theory (Taylor and Francis, London, 1994).

26. Salamon, M. B. \& Jaime, M. The physics of manganites: Structure and transport. Rev. Mod. Phys. 73, 583-628 (2001).

27. Maiser, E. et al. Pulsed-laser deposition of $\mathrm{Pr}_{2-x} \mathrm{Ce}_{x} \mathrm{CuO}_{4-y}$ thin films and the effect of high-temperature post-annealing. Physica C: Supercond. 297, 15-22 (1998).

28. Onose, Y., Taguchi, Y., Ishizaka, K. \& Tokura, Y. Charge dynamics in underdoped $\mathrm{Nd}_{2-x} \mathrm{Ce}_{x} \mathrm{CuO}_{4}$ Pseudogap and related phenomena. Phys. Rev. B 69, 024504 (2004).

29. Kempa, H., Esquinazi, P. \& Kopelevich, Y. Field-induced metal-insulator transition in the c-axi resistivity of graphite. Phys. Rev. B 65, 241101 (2002).

30. Singh-Bhalla, G., Du, X. \& Hebard, A. F. Contribution of interface capacitance to the electric field breakdown in thin-film Al-AlOx-Al capacitors. Appl. Phys. Lett. 83, 2417-2420 (2003).

\section{Acknowledgements}

We thank E. Dagotto, P. Littlewood and S. Obukhov for useful discussions. This work was supported by the National Science Foundation under Grant No. 0404962.

Correspondence and requests for materials should be addressed to A.F.H.

Supplementary Information accompanies this paper on www.nature.com/naturephysics.

Competing financial interests

The authors declare no competing financial interests.

Reprints and permission information is available online at http://npg.nature.com/reprintsandpermissions/ 